\title{
CONDICIONES PARA LA IMPLEMENTACIÓN DE UN MARCO REGULATORIO AL USO DE RPAS EN HONDURAS
}

\author{
Omri Amaya ${ }^{1, *}$, Ramón Bueso ${ }^{1,+}$, Lenin Valeriano ${ }^{2, \ddagger}$ Allister Stefan ${ }^{3, \S}$ \\ ${ }^{1}$ Departamento de Ciencias Aeronáuticas, Facultad de Ciencias Espaciales, UNAH \\ ${ }^{2}$ Agencia Hondureña de Aeronáutica Civil \\ ${ }^{3}$ Ik'Unmanned Aerial Solutions
}

Recibido: $1 /$ noviembre/2019

Aceptado: 20/noviembre/2019

DOI: https://doi.org/10.5377/ce.v12i1.9638

\begin{abstract}
RESUMEN
El presente artículo resume los resultados de una investigación que tuvo como objeto de estudio conocer las Condiciones para la Implementación de un Marco Regulatorio al Uso de Sistemas de Aeronaves No Tripuladas (RPAS) en Honduras. La investigación aplica un proceso de Investigación - Acción en el cual se involucraron la Agencia Hondureña de Aeronáutica Civil (entidad regulatoria) y la Universidad Nacional Autónoma de Honduras como entidades regulatoria y de investigación respectivamente. La dinámica permitió elaborar una planificación en donde los avances de la investigación se han utilizado para la modificación de la misma planificación con la mediación de consensos entre las partes, las cuales se han vuelto investigadoras y ejecutoras de su propia planificación.

La normativa de RPAS resultante del proceso de investigación presentado debe tomar en cuenta la seguridad operacional en el uso masivo de estos artefactos, así como sus distintas aplicaciones que van del ámbito recreativo al del trabajo y negocios, pasando por el amplio campo de la investigación científica.

La Agencia Hondureña de Aeronáutica Civil (AHAC) es el ente mediador del espacio aéreo nacional y coordina la reglamentación nacional, para conceptualizar e implantar una normativa sobre RPAS, convirtiendo este reto en una oportunidad de apoyo interinstitucional por parte de la UNAH a través del Departamento de Ciencias Aeronáuticas (DCA), adscrita a la Facultad de Ciencias Espaciales (FACES).

Palabras clave: Sistemas de aeronaves no tripuladas (RPAS), dron, Departamento de Ciencias Aeronáuticas DCA, Agencia Hondureña de Aeronáutica Civil (AHAC), aeromodelismo, aeropuerto.
\end{abstract}

\begin{abstract}
This article presents the results of an investigation that had as an object of study to know the Conditions for the Implementation of a Regulatory Framework for the Use of Unmanned Aircraft Systems (UAS) in Honduras. The research applies a Research - Action process in which the Honduran Civil Aviation Agency (regulatory entity) and the National Autonomous University of Honduras were involved as academic and research entities respectively. The dynamics allowed to elaborate a planning where the
\end{abstract}

*omri.amaya@unah.edu.hn

†ramon.bueso@unah.edu.hn

${ }^{\ddagger}$ lvaleriano@ahac.gob.hn

sinfo@ik-uas.com 
advances of the investigation have been used for the modification of the same planning with the mediation of consensus between the parties, which have become investigators and executors of their own planning.

The RPAS regulations resulting from the research process presented must take into account safety in the mass use of these devices, as well as their different applications ranging from recreational to work and business, through the broad field of scientific research.

The Honduran Civil Aviation Agency (AHAC) is the mediator of the national airspace and coordinated the national regulations, to conceptualize and implement regulations on UAS, making this challenge an opportunity for interagency support by UNAH through the Department of Aeronautical Sciences (DCA), attached to the Faculty of Space Sciences (FACES).

Keywords: Remotely Piloted Aircraft (RPAS), drone, DCA Department of Aeronautical Sciences, Honduran Civil Aviation Agency (AHAC), aeromodelling, airport.

\section{Introducción}

Los Sistemas de Aeronave Pilotada a Distancia RPAS conocidos como drones, han evolucionado de una manera acelerada con el impulso de las nuevas tecnologías; con la irrupción de la tecnología y la masificación de uso de RPAS se vuelve un desafío el cumplimiento con los estándares de seguridad que garanticen a los usuarios y al público en general la prevención de incidentes y accidentes.

La investigación abordada en este artículo buscó apoyar el rol que le corresponde a la Agencia Hondureña de Aeronáutica Civil (AHAC) como ente regulador de la aeronáutica en Honduras, específicamente en el establecimiento de un marco regulatorio para el uso y aplicación de los artefactos denominados RPAS comúnmente conocidos como drones. La instauración de estas regulaciones tiene básicamente dos grandes momentos, primero la elaboración y aplicación de un instrumento normativo denominado Circular de Obligatorio Cumplimiento (COC), con el cual se establecen parámetros de uso y aplicación transitorios y en un segundo momento se diseña un instrumento de aplicación permanente, conocido como Regulación de Aeronáutica Civil, incorporando elementos que atienden un amplio espectro de indicaciones, pautas y restricciones para los usuarios de RPAS, garantizando la seguridad operacional en distintos sentidos y capitalizando las lecciones aprendidas durante el tiempo de aplicación de la COC.

\section{Metodología}

\subsection{Temporalidad y enfoque}

La investigación se desarrolló desde Enero a Diciembre del 2018 y aplicó una metodología de Investigación - Acción Participativa (IAP), puesto que la información recogida por los investigadores procedente de un equipo más amplio compuesto por representantes de la UNAH y la AHAC, alimentó una dinámica de planificación en la cual de forma dialéctica se hicieron correcciones sobre la marcha a las acciones de la misma planificación, generando de esta manera un espiral de pasos sucesivos en los que se investiga al mismo tiempo que se interviene (Montero y León, 2002).

\subsection{Tipo de diseño}

Se siguió un diseño participativo en el cual se involucró "a los miembros del grupo o comunidad en todo el proceso de estudio (desde el planteamiento del problema hasta la elaboración del reporte) y la implementación de acciones..." (Sampieri et al., 2010). La lógica participativa representa un proceso por medio 
del cual los sujetos investigados, en la práctica, son auténticos coinvestigadores, participando activamente en el problema a ser investigado, la información obtenida, los métodos y técnicas a ser utilizados, el análisis e interpretación de los resultados, la decisión de qué hacer con los resultados y las acciones que se programaran en el futuro, conjugando los conocimientos del investigador con los conocimientos prácticos y vivencias de los participantes.

\subsection{Aplicación de la metodología IAP}

La aplicación de la metodología IAP al ámbito aeronáutico en materia de cooperación y asesoría es la primera iniciativa en este campo en nuestro país. Si bien la línea de tiempo de la investigación reportada se extiende de enero a diciembre de 2018 la investigación continúa y este lapso de tiempo se inscribe en un período mayor, el cual se puede dar por satisfecho cuando en Honduras se cuente con una Regulación de Aeronáutica Civil para RPAS. En base a este horizonte se ha reelaborado la planificación y se han establecido de manera consensuada las distintas fases del proceso.

Las etapas y fases de la Investigación Acción Participativa (IAP) para todo el proceso son las siguientes:

Tabla 1: Etapas y fases de la investigación acción participativa. Fuente: Montero y León (2002).

Etapa de pre-investigación: síntomas, demanda y elaboración del proyecto.

0 . Detección de la necesidad de una normativa sobre RPAS basados en experiencias de otros países.

1. Eventos de Capacitación para diagnosticar la necesidad de la normativa.

2. Planteamiento de la investigación a través de la creación de un grupo de trabajo interinstitucional UNAH-AHAC.

\section{Primera etapa. Diagnóstico.}

Conocimiento contextual del campo aeronáutico y las normas y regulaciones establecidas

para su organización y de esa forma definir la problemática a partir de la documentación existente

y de entrevistas a expertos institucionales e internacionales.

3. Comisión de seguimiento constituida

4. Documentación técnica y legal consultada como marco normativo

5. Esquema del documento como un marco normativo.

6. Proceso de diseño y socialización del proyecto para analizar las reacciones y sugerencias de grupos para afinar detalles de la normativa propuesta. 7. Primera propuesta de normativa.

\section{Segunda etapa. Programación.}

Proceso de apertura a todos los conocimientos y puntos de vista existentes, utilizando métodos cualitativos y participativos.

8. Mesas de trabajo (entrevistas grupales a la base social).

9. Análisis de los resultados de las mesas de trabajo.

10. Entrega y discusión de la segunda propuesta de normativa.

11. Realización de talleres a personal involucrado

\section{Tercera etapa. Conclusiones y propuestas}

Negociación y elaboración de propuestas concretas.

12. Construcción del programa de socialización de la normativa al público involucrado.

13. Elaboración y entrega del informe final

Etapa post-investigación: puesta en práctica del programa de socialización y evaluación. Nuevos síntomas 


\subsection{Detección de síntomas y elaboración de proyecto.}

El antecedente principal de los trabajos sobre RPAS del DCA es la investigación finalizada en Julio del 2017 "Control autónomo de sistemas aéreos No Tripulados Tipo Cuadricóptero" (Carías, 2016). En este documento se declara que «Honduras, como signatario de Convenio de Chicago, y miembro activo de la Organización de la Aviación Civil Internacional, ya posee el borrador de la Regulación de Aeronáutica Civil denominada "Regulación de Aeronaves No Tripuladas, Aeronaves de Modelismo y Fuegos Artificiales». Esta legislación todavía pendiente de aprobación obedece a la gran afluencia de este tipo de vehículos en el espacio aéreo hondureño; sin embargo, el avance de la dimensión legislativa no se corresponde con el conocimiento, análisis y desarrollo de la parte técnica" (Carías, 2016). Otro referente para el DCA en materia de RPAS fue una jornada realizada en España en 2016 en donde se presentó un estado del arte del uso y aplicación de los RPAS a nivel mundial, la visión reportada en este evento permitió encuadrar el caso de Honduras en un panorama mundial logrando identificar grandes vacíos que deben ser atendidos.

Bajo las experiencias descritas en el inciso anterior, el Departamento de Ciencias Aeronáuticas impartió el Seminario: "Introducción a Sistemas Aéreos No Tripulados de Operación Remota" (DCA, 2017), del cual se desarrollaron dos ediciones con la siguiente asistencia:

Tabla 2: Fechas de los eventos de capacitación a personal de la AHAC por parte de la UNAH.

\begin{tabular}{ccc}
\hline $\mathrm{N}^{\circ}$. Edición & Fecha & $\mathrm{N}^{\circ}$. de participantes \\
\hline Primera (ver figura 1) & 31 de julio del 2017 & 18 \\
Segunda (ver figura 2) & 23 de octubre del 2017 & 16 \\
\hline
\end{tabular}

En dichos eventos (UNAH, 2017) la AHAC confirmó que las capacitaciones en la temática y la normativa para la operación de RPAS en Honduras no existían, y que lo descrito en el RAC 02 "Reglas del Aire" mostraba "vacíos" que generaban algún tipo de confusión, ya que no se podía dar respuesta a solicitudes de operadores ni a denuncias de "drones" vistos cerca de la operación de aeronaves tripuladas.

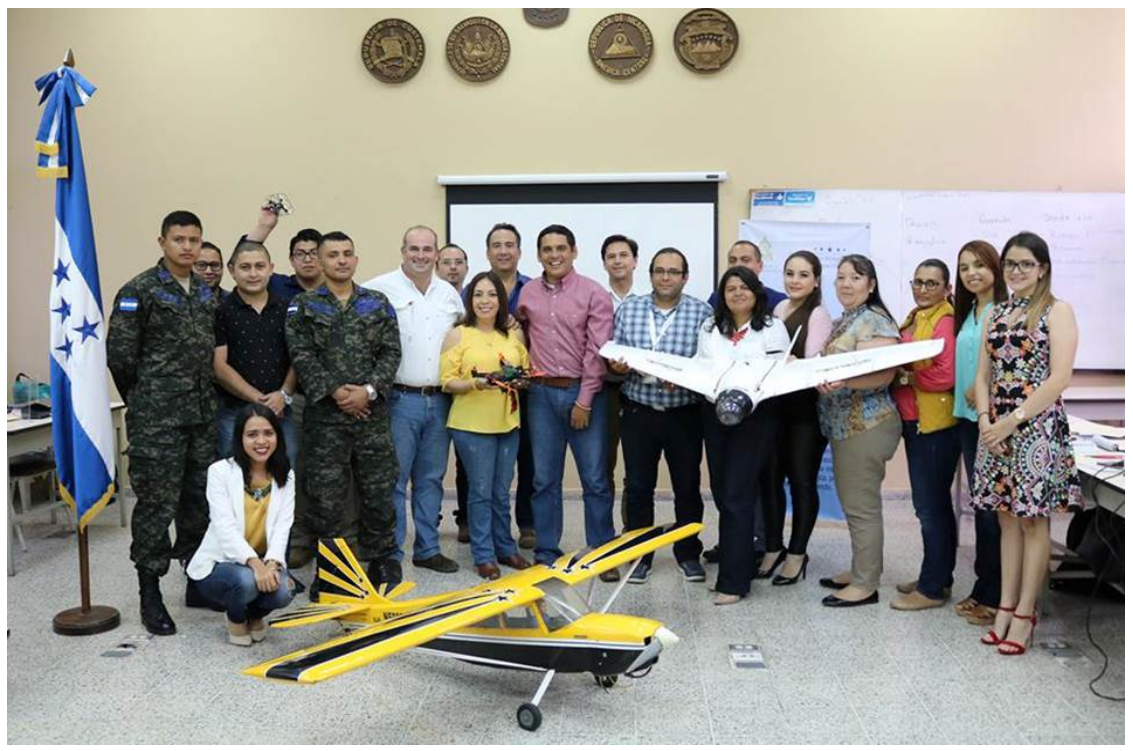

Figura 1: Primer grupo capacitado en el proceso de incorporación de una normativa en RPAS en Honduras. 


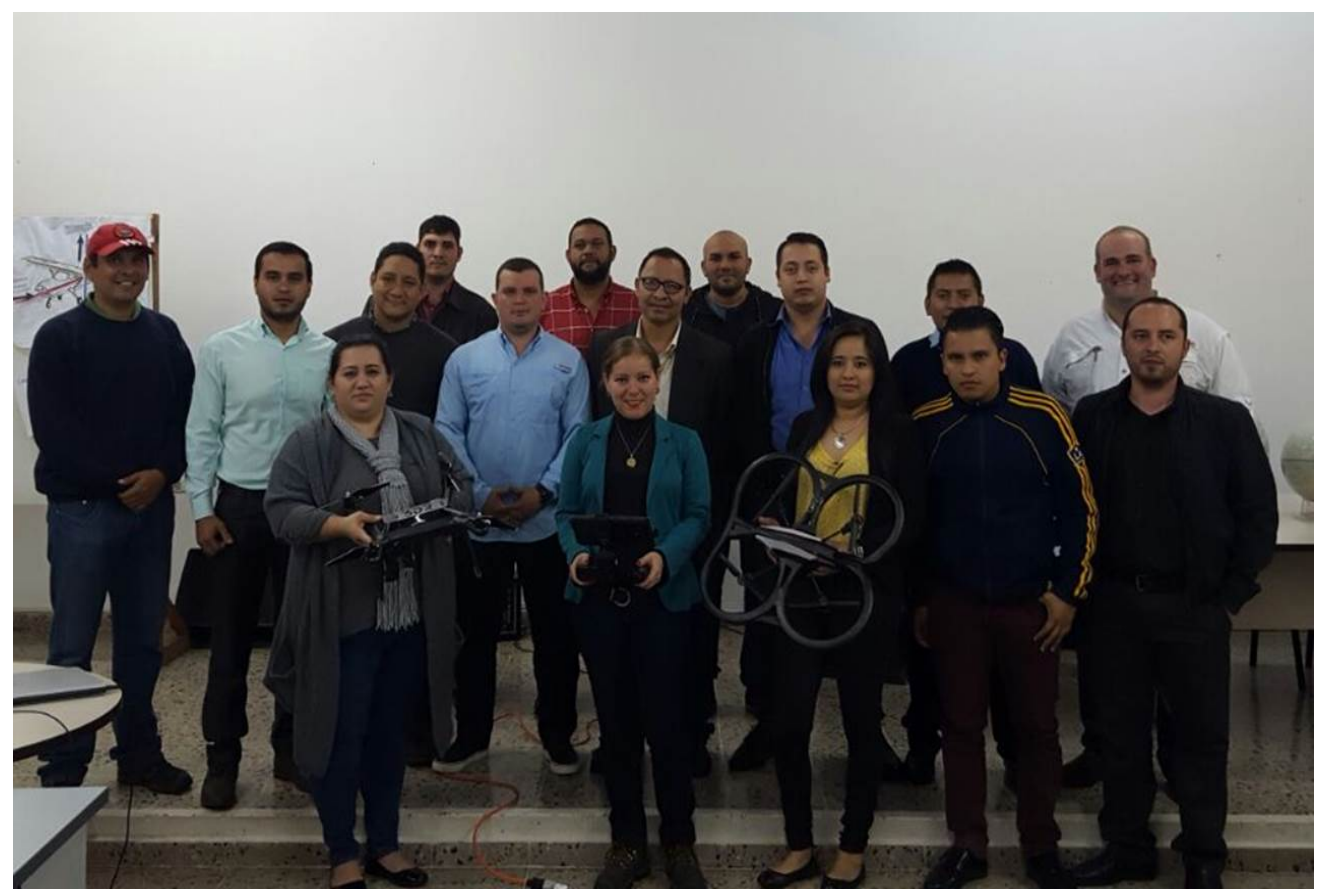

Figura 2: Segundo grupo capacitado en el proceso de incorporación de una normativa en RPAS en Honduras.

En vista de lo relatado, la UNAH por medio del DCA desarrolló una reunión con personal de la AHAC para plantearse el proceso a seguir para conformar un equipo de trabajo interinstitucional que redactara la normativa sobre RPAS.

\section{Resultados}

\subsection{Imperativos de la legislación hondureña y orientaciones OACI}

La ley de Aeronáutica Civil de Honduras aprobada en 2017, indica entre sus finalidades "promover el desarrollo nacional apoyando y fomentando la realización de las actividades vinculadas al subsector de aeronáutica civil, creando las condiciones necesarias y ejecutando acciones administrativas, técnicas y operacionales de regulación y promoción para operar servicios de transporte aéreo interno e internacional en forma ordenada, segura, eficiente y confiable..." a tenor con dicha ley, le corresponde a la AHAC proponer una opción normativa no solo para establecer parámetros y requisitos para la operación de los RPAS, sino también para la creación de espacios para entrenamiento, la promoción de certificación de centros de instrucción aeronáuticos con programas de entrenamiento aprobados, la promoción de certificación de operadores comerciales de RPAS y el incentivo de actividades de motivación de las buenas prácticas del uso de RPAS en el espacio aéreo hondureño; estos cometidos se pueden lograr apoyando la creación de centros instrucción aeronáutica, clubes aéreos, museos, centros de educación superior y de investigación aérea pública y privada en el ámbito de los RPAS, todo en aras de la seguridad operacional. Por otra parte, dado que nuestra nación es miembro de la Organización de Aviación Civil Internacional (OACI), se procedió a seguir los lineamientos que este organismo establece como propuesta para una correcta implementación de una normativa que aborde el uso de los RPAS en el país. Dicha implementación responde a un proceso que se resume en el siguiente gráfico (ver figura 3). 


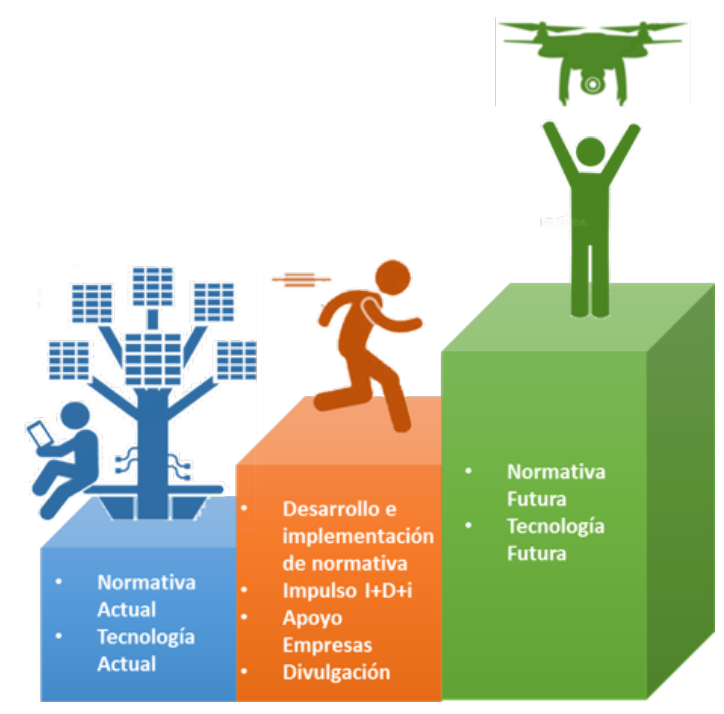

Figura 3: Elemento esenciales a tomar en cuenta en el proceso de implementación de una normativa en RPAS en un estado miembro de OACI.

A continuación, las recomendaciones generales por parte de la OACI en el proceso de implementación de una normativa de RPAS (OACI, 2019):

\section{a. Comprensión de la situación}

La OACI les confirma a las autoridades de aviación civil que estas tienen la responsabilidad de garantizar la seguridad operacional de la aviación y proteger al público de los peligros en la aviación.

i. Los RPAS pueden utilizarse como juguetes o ser diseñadas para usuarios profesionales, con pesos que varían desde decenas de gramos hasta miles de kilogramos.

ii. Las RPAS pueden transportar carga y estar equipadas con cámaras y otros sensores p. ej., fotodetección y telemetría (LIDAR) o infrarrojos.

iii. Los modos de operación pueden variar desde operaciones con visibilidad directa visual (VLOS) y operaciones más allá de la visibilidad directa (BVLOS) hasta operaciones de bandadas de dispositivos automáticos en red que vuelan en formación.

b. ¿Para qué se utilizan?

Como entidad que reglamenta, es fundamental reconocer los beneficios sociales que aportan los RPAS y la necesidad de facilitar operaciones de manera segura e incluir: actividades humanitarias, búsqueda y salvamento, extinción de incendios, supervisión de infraestructuras, así como investigación y desarrollo $(I+D)$. Los beneficios económicos de los RPAS son igualmente importantes; no obstante, independientemente de sus beneficios sociales y económicos, su uso puede plantear problemas de seguridad operacional que la entidad que reglamenta debe tener en cuenta. El uso recreativo de los RPAS puede generar aún más inquietudes.

c. ¿Dónde vuelan? 
El entorno físico de cada estado es diferente y la entidad que reglamenta tiene que tener en cuenta los emplazamientos operacionales de los RPAS. Dicha entidad tiene que responder a la demanda de operar UAS teniendo en cuenta los siguientes aspectos relativos al entorno físico:

i. La proximidad de aeródromos, espacios aéreos controlados o rutas aéreas de mucho movimiento. Por ejemplo, Singapur opera en espacio aéreo controlado con tráfico aéreo muy activo,

ii. Zonas urbanizadas/densamente pobladas versus zonas rurales/abiertas. Por ejemplo París o la ciudad de Nueva York City versus campos agrícolas o el interior de Australia,

iii. La conveniencia de operar RPAS cerca de parques nacionales, zonas de acceso restringido/prohibido y zonas delicadas,

d. ¿Quiénes los operan?

i. Personas particulares y organizaciones compuestas por una sola persona,

ii. Organizaciones de tamaño pequeño o mediano, incluidas las de aficionados,

iii. Organizaciones multinacionales,

Otras consideraciones y recomendaciones:

La entidad que reglamenta debería determinar áreas de interés clave y formular reglamentos que puedan aplicarse eficazmente. Los reglamentos de un Estado deberían abarcar todas las operaciones de RPAS en el espacio aéreo interior de modo que el marco normativo que se establezca sea compatible con los reglamentos aeronáuticos existentes y con los reglamentos de otros sectores. Esto debería incluir:

i. Espectro de frecuencias: muchos estados reglamentan las bandas de frecuencias que un producto podría utilizar, esto incluiría el enlace de mando y control (C2),

ii. Transporte de mercancías peligrosas y otros materiales peligrosos p. ej., muestras médicas,

iii. Derechos de los propietarios de terrenos e inmuebles, reglas sobre la intrusión ilegal en propiedades privadas y respeto de la vida privada,

iv. Códigos penales,

v. Asuntos relativos a aduanas e inmigración,

vi. Seguridad operacional del producto-la legislación relativa a la seguridad operacional de los productos puede aplicarse a los RPAS fabricados como juguetes/dispositivos electrónicos.

\subsection{Comisión de seguimiento constituida}

El comité conformado para la elaboración de una regulación para RPAS en respuesta a la situación específica de Honduras, tuvo como función el establecer los requerimientos para el uso de RPAS, con un enfoque basado en pruebas e incremental que puede ser beneficioso para una industria como la hondureña que no está madura y que podría evolucionar de manera diferente de lo esperado por la autoridad de aviación civil; es ahí donde un proceso ordenado es fundamental. 


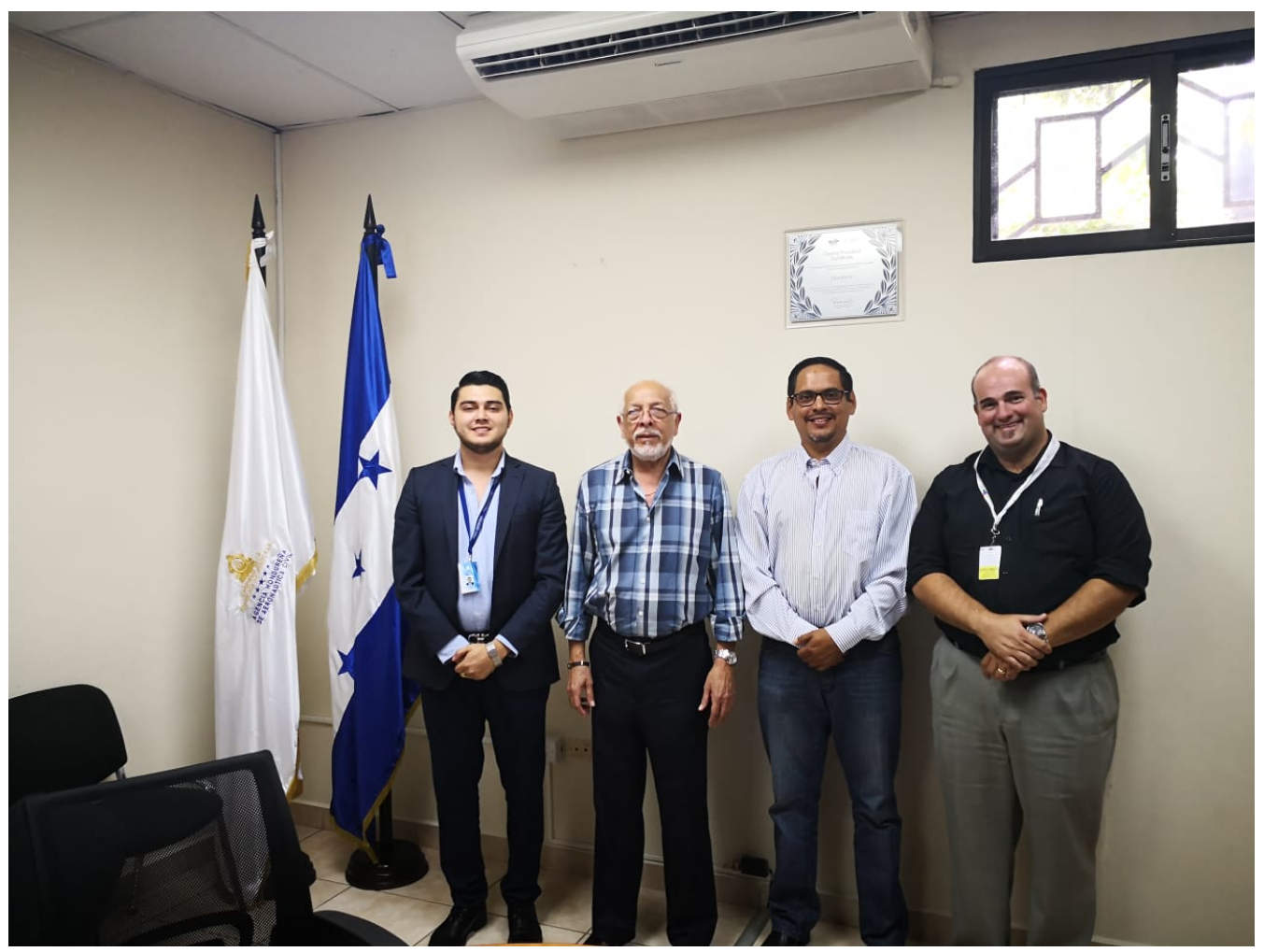

Figura 4: Comisión de seguimiento de normativa de RPAS en Honduras.

Dicho comité inicio funciones en febrero del 2018 y cuenta con miembros de la UNAH y de AHAC (ver figura 4). Dicho comité inicio funciones posteriores a la reunión del 5 de febrero del 2018, siendo sus miembros el Abogado Lenin Valeriano por parte de la AHAC, el Master Omri Amaya por parte de la UNAH y el Doctor Allister Stefan como experto en RPAS.

\subsection{Elementos de una regulación típica}

Para la selección de marcos regulatorios a consultar se tomaron en cuenta los siguientes criterios: se priorizo la normativa con mayor tiempo de aplicación y que el país donde se aplicaran fuera del ámbito latinoamericano; además se examinaron los casos en los que la normativa tuvo niveles relevantes de rechazo por parte de la comunidad de operadores y en donde esta comunidad hizo saber sus quejas a la Autoridad Aeronáutica; tal es el caso de Costa Rica, en donde los medios publicaron encabezados como: "Polémico reglamento para uso de drones entró en vigencia este martes" de (Monumental, 2018) y otros como: "Operadores de drones desconformes con nueva regulación" del diario La República (República, 2018); por lo cual el comité busco asesoría en otras entidades de países amigos. Aplicando los criterios antes mencionados se tomaron como referencias las normativas siguientes:

i. Circular Reglamentaria $N^{\circ} .002$ de Colombia (Aerocivil, 2015).

ii. Circular Obligatoria que Establece los Requerimientos para Operar un Sistema de Aeronave Pilotada a Distancia (RPAS) en el Espacio Aéreo Mexicano (de Aeronáutica Civil de México, 2017).

iii. Regulación de los Vehículos Aéreos No Tripulados de El Salvador (de Aviación Civil, 2018).

Es de resaltar en la normativa consultada, la claridad con la que expresan los requerimientos a los operadores RPAS de sus estados, así como que su implementación se ha desarrollado de manera progresiva. Ya 
teniendo estas normativas como formato para establecer los estándares mínimos del cuerpo de contenido, se integraron las consideraciones propuestas por la OACI para definir los requerimientos a establecer en la regulación que se proponga. Específicamente se tomó en cuenta la Guía Práctica OACI sobre los UAS (OACI, 2019), en donde metodológicamente, se describen tópicos que complementan cada uno de los elementos que la reglamentación debía abordar.

Dichos elementos son presentados en la siguiente figura:
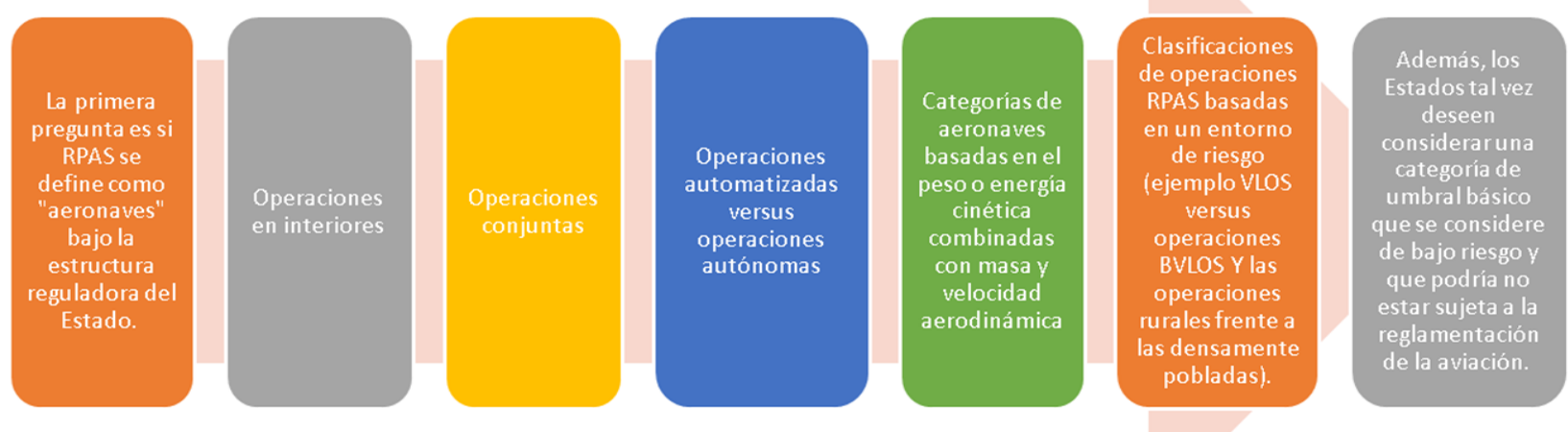

Figura 5: Elemento esenciales a tomar en cuenta en el proceso de implementación de una normativa en RPAS en un estado.

Ya definidos los elementos fundamentales que formarían parte de la normativa, se procedió a la evaluación consensuada de qué figura legal debería ser utilizada para este primer paso de la legalización de la operación de drones en el espacio aéreo hondureño. Es así que se propone la figura Circular de Obligatorio Cumplimiento, desde la cual se pueden abordar los elementos esenciales de una normativa, conservando la condición de documento experimental que permite recoger información para la elaboración posterior de un RAC definitivo.

\section{Discusión}

La socialización/consulta tuvo como fin el presentar tanto elementos del proceso de creación de la normativa, así como un enfoque de lo que la irrupción de la tecnología RPAS significa para la es y, sus limitaciones y ventajas tanto a nivel de características de vuelo como de aplicaciones a diferentes ámbitos tanto recreativos como comerciales. Para las consultas hechas a distintos públicos se preparó un documento de Circular de Obligatorio cumplimiento (COC) tomando como principal referente el Reglamento No. 002 de Colombia; a continuación se resumen los principales aportes de las consultas hechas.

\section{a. Reunión con experto en navegación aérea AHAC}

El Departamento de Navegación Aérea de la AHAC, encabezado por el Ingeniero Heriberto Sierra, destacó los riesgos provocados por el vuelo de drones en las servidumbres aeronáuticas, confirmándonos la necesidad de establecer perímetros alrededor de dichas infraestructuras, de manera que disminuya lo más posible el riesgo de colisión entre aeronaves tripuladas y RPAS. 


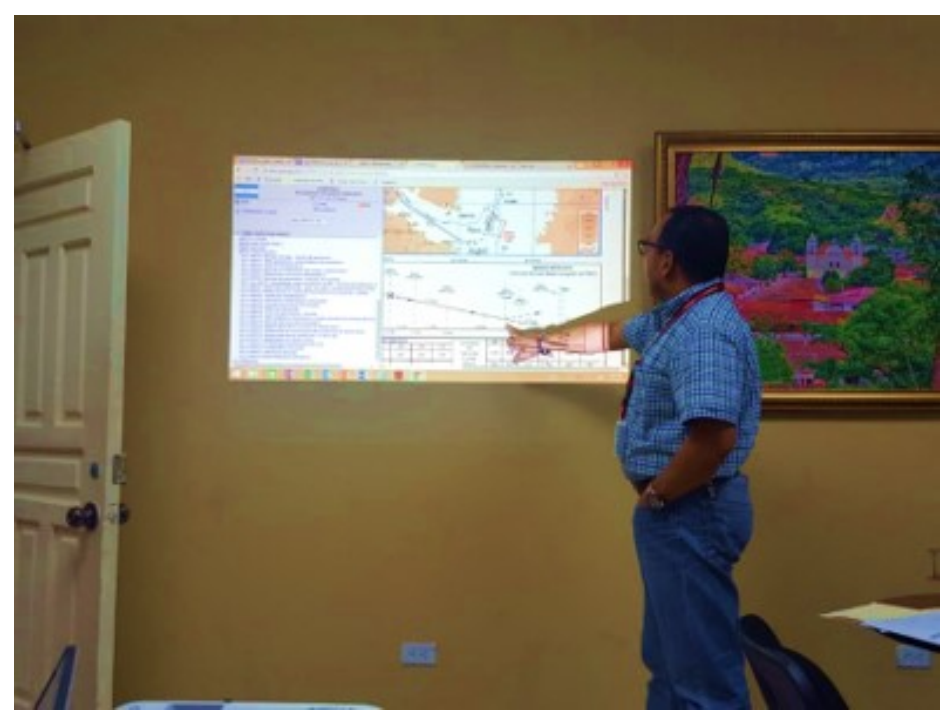

Figura 6: Demostración por parte del Ingeniero Sierra haciendo uso del AIP de las zonas críticas aledañas a los aeródromos en Honduras.

La fuente principal para el análisis por parte de Navegación Aérea fue el documento electrónico conocido como AIP (Publicación de Información Aeronáutica por sus siglas en ingles), en el cual se pueden visualizar los diferentes aeropuertos internacionales, así como los aeródromos y helipuertos oficiales por parte de la $\mathrm{AHAC}$, su posición geográfica, dimensiones y características principales, determinándose de esta manera que dada su categoría, cada tipo de RPAS deberá tener un perímetro diferente para el acercamiento a las áreas de operaciones de aeronaves tripuladas (AHAC, 2019).

\section{a. Primer seminario regional en navegación aérea}

En la Exposición N 11 denominada "Panel de Expertos: Retos de la Integración de los RPAS al Espacio Aéreo Hondureño" se concluyó que no todos los usos de RPAS representan un desafío para la aeronáutica que se desarrolla en aeródromos y aeropuertos en términos de invasión de los espacios utilizados; sin embargo, al considerar todo el espectro de diseños, pesos y sistemas de control, usos y aplicaciones, se vio que existen muchas circunstancias en las que se da conflictividad entre los RPAS y las aeronaves. El momento por el que pasa Honduras de gran auge de vehículos aéreos no tripulados tanto por su masiva comercialización como por los múltiples usos que se les está dando, es más que oportuno para generar una regulación que establezca las limitaciones necesarias para incorporar estos artefactos (DCA, 2018).

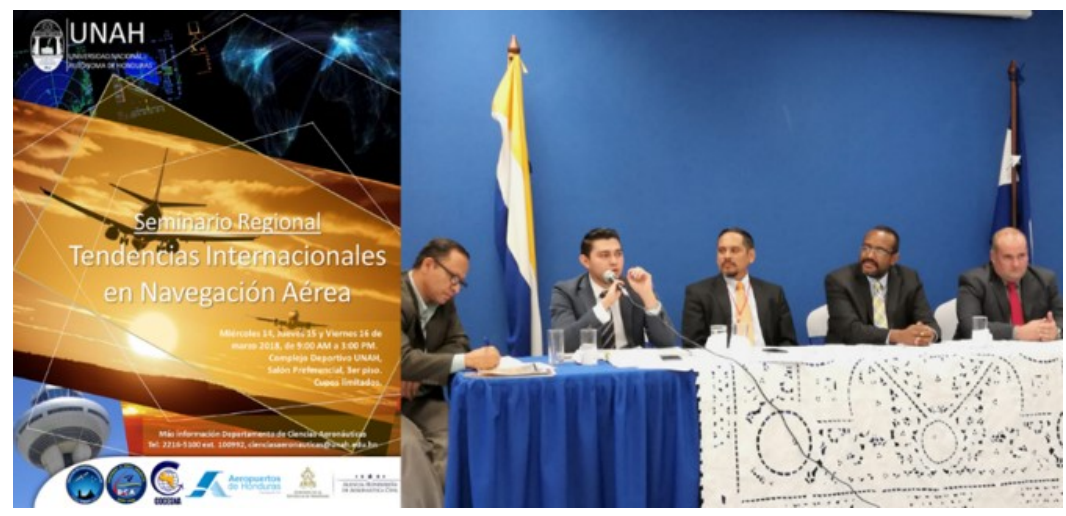

Figura 7: Poster publicitario e integrantes de mesa de trabajo en el I Seminario de Navegación Aérea del DCA. 


\section{b. XII Congreso de Investigación Científica, UNAH 2018}

En el XII Congreso de Investigación Científica de la UNAH (ver figura 8) se contó con la presentación de un diseño para un uso específico de RPAS denominado "Desarrollo de un Sistema de UAV para la determinación de flujos turbulentos atmosféricos"; la cual fue brindada por el Doctor Oscar Garibaldi, quien es docente e investigador de la Universidad Tecnológica. Su ponencia proporciono los razonamientos para el desarrollo de la Normativa de RPAS en lo correspondiente a la innovación, investigación y desarrollo.

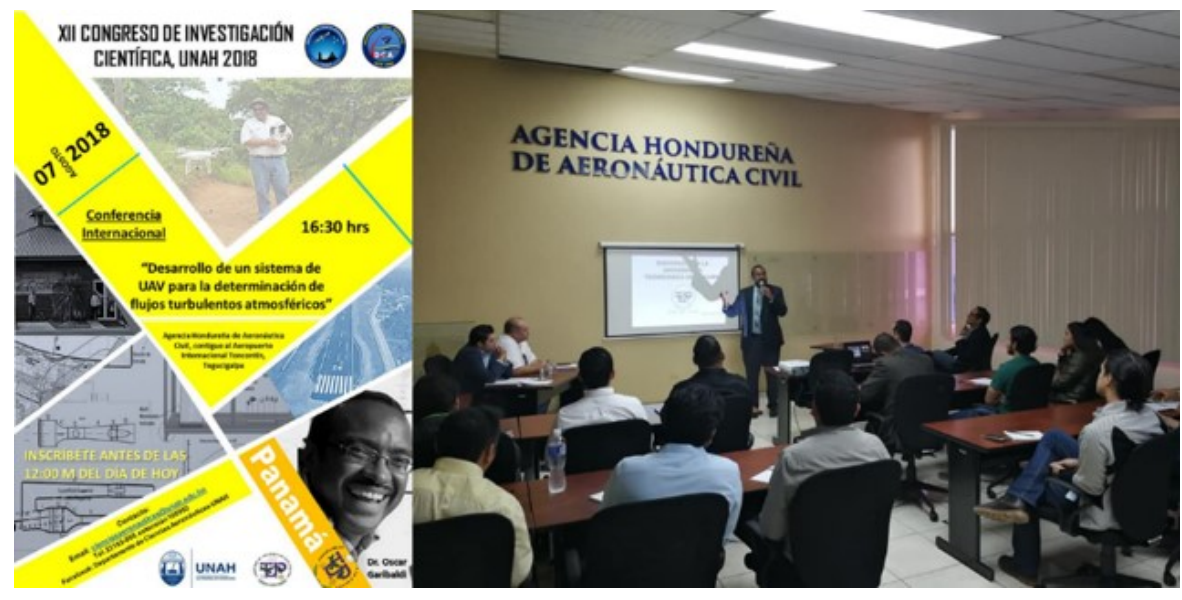

Figura 8: Poster publicitario del XII Congreso de Investigación Científica de la UNAH y foto de la ponencia del Doctor Oscar Garibaldi.

\section{c. Reuniones con las Fuerzas Armadas}

En las dos reuniones sostenidas con las Fuerzas Armadas (ver figura 9) se hizo ver la perspectiva que las entidades de Defensa tienen acerca de estos dispositivos, lo que puso de manifiesto la necesidad de incorporar en la normativa componentes que garanticen aquellos aspectos de la seguridad nacional que están en manos de esta Dependencia del Estado. Las actividades realizadas se resumen en la siguiente tabla:

Tabla 3: Fechas de los eventos de capacitación a personal de la AHAC por parte de la UNAH.

\begin{tabular}{lcccc}
\hline Item. & Fecha & Tema & Lugar & $\mathbf{N}^{\circ}$. de participantes \\
\hline Socialización & 06 de julio del 2018 & $\begin{array}{l}\text { Conferencia RPAS a miembros } \\
\text { de la Fuerza Aérea Hondureña }\end{array}$ & FAH & 10 \\
Socialización & 20 de noviembre del 2018 & $\begin{array}{l}\text { Conferencia RPAS a miembros } \\
\text { de la Fuerza Aérea Hondureña }\end{array}$ & FAH & 15 \\
\hline
\end{tabular}


Las siguientes fotografías fueron tomadas durante las actividades realizadas:
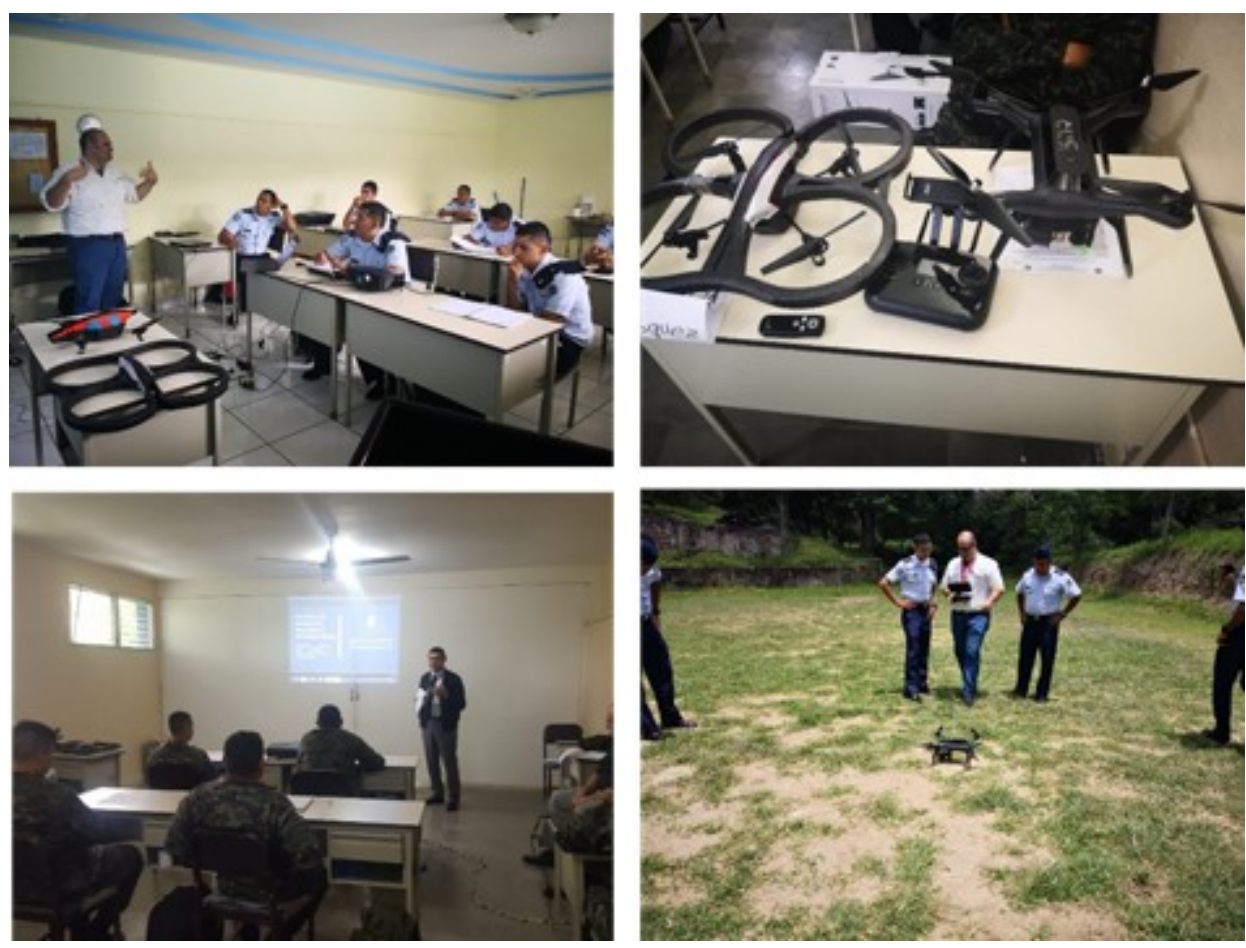

Figura 9: Actividades de capacitación a personal de la Fuerza Aérea Hondureña.

Primera propuesta de normativa

El Borrador de la Normativa, presentada para la validación por parte de la sociedad hondureña, fue denominada: Circular de Obligatorio Cumplimiento, "REGISTRO DE OPERADORES Y LIMITACIONES DE OPERACIÓN DE SISTEMAS DE AERONAVES PILOTADAS A DISTANCIA (RPAS)", Numero COCATL-002-2018, se presentó con el siguiente contenido:

- Denominación

- Objetivo

- Normativa de referencia

- Aplicabilidad

- Base jurídica:

- Definiciones y abreviaturas:

- Clasificación:

- Limitaciones de operación de "RPAS" en el espacio aéreo hondureño.

a. Requerimientos y limitaciones generales para todas las categorías:

b. Requerimientos y limitaciones de operaciones para uso comercial e institucional/gubernamental: 
- Autorizaciones especiales de operación (excepciones):

- Zonas autorizadas para actividades recreativas y áreas de prueba para desarrollo tecnológico

- Distribuidores y fabricantes

- Procedimiento y requisitos para el registro e identificación de sistemas de aeronaves pilotadas a distancia (RPAS)

- Aspectos de inscripción

- Procedimiento y requisitos para registro de piloto remoto de RPAS

- Requisitos mínimos piloto remoto/observador:

- Solicitud de operación RPAS comercial:

- Solicitud de operación para uso institucional/gubernamental

- Procedimiento y requerimientos para la emisión de autorizaciones especiales:

- Requisitos

- Vigencia de los permisos

- Disposiciones sancionatorias/casos de aplicación y procedimiento:

- Fecha de efectividad

- Anexos

- Anexo 1: Formulario de registro RPAS

- Anexo 2: Registro de piloto remoto/Observador RPAS

- Anexo 3: Solicitud para operaciones comerciales RPAS

- Anexo 4: Solicitud para operaciones especiales (Exenciones)

- Anexo 5: Cuerpo de conocimientos para piloto remoto/Observador

- Anexo 6: Contenido del manual del operador

\section{Conclusiones}

- La investigación constata la urgencia de contar en Honduras con una regulación que reglamente desde la Agencia Hondureña de Aeronáutica Civil el campo de uso y aplicación de los RPAS, el cual en la actualidad está experimentando un enorme crecimiento.

- Para la presente investigación la UNAH ha puesto a la disposición de la Autoridad de la Aeronáutica Civil Hondureña una metodología de investigación que ya ha sido aplicada a diversas temáticas de la aeronáutica civil generando productos tangibles; es de esperarse que los resultados de la presente investigación no solo sean concretos, sino que también se apliquen con la celeridad que se requiere. 
- Una de las garantías de aplicación de los resultados obtenidos es el equipo que ya fue constituido para la presente investigación, tanto la Agencia Hondureña de Aeronáutica Civil como la UNAH son voces autorizadas para elaborar una propuesta de regulación que sobre la base del rigor científico ponga orden en la incorporación de los RPAS a los espacios aéreos nacionales.

- Un plan estratégico para la elaboración y aplicación de un RAC aplicables al uso y aplicación de RPAS comporta al menos cinco momentos: 1) un estudio de estado del arte sobre la regulación de RPAS; 2) la redacción y aplicación una circular de obligatorio cumplimiento; 3) la elaboración de un RAC para RPAS; 4) la divulgación masiva de esta regulación y de las indicaciones de uso y aplicación de RPAS; 5) la aplicación de la regulación; hasta ahora se ha trabajado hasta el segundo momento.

- A todo este proceso debe dársele seguimiento con al menos tres actores: la AHAC, la UNAH y los usuarios de RPAS.

\section{Siglas utilizadas}

AHAC: Agencia Hondureña de Aeronáutica Civil

BVLOS: Más allá de la visibilidad directa del piloto (por su nombre en inglés Beyond Visual Line of Sight).

COC: Circular de Obligatorio Cumplimiento.

DCA: Departamento de Ciencias Aeronáuticas.

I+D+i: Innovación más Desarrollo más Investigación.

LIDAR: Dispositivo que permite determinar la distancia desde un emisor láser a un objeto o superficie utilizando un haz láser pulsado (por sus siglas en inglés Laser Imaging Detection and Ranging)

RPAS: Vehículo aéreo no tripulado (por sus siglas en inglés Remotely Piloted Aircraft System).

UNAH: Universidad Nacional Autónoma de Honduras.

VLOS: Al alcance de la visibilidad externa del piloto (por sus siglas en inglés Visual Line of Sigth).

\section{$7 \quad$ Referencias}

Aerocivil (2015). Circular reglamentaria $N^{\circ} .002$ «operadores de drones disconformes con nueva regulación». Bogota.

AHAC (2019). Archivo situacionista hispano. http://www.ahac.gob.hn/AIPMH_AMDT_0519/AIP_ 1905/Eurocontrol/HONDURAS/20190620AIRAC/html/indexesES.html.

Carías, O. A. A. (2016). Control autónomo de sistemas aéreos no tripulados tipo cuadricóptero. Tegucigalpa: DCA.

DCA (2017). Informe de seminario introducción a sistemas aéreos no tripulados de operación remota (RPAS). Documento no publicado. 
DCA (2018). Informe final proyecto "necesidad de formación de profesionales en el área de navegación aérea a nivel superior en honduras a mayo 2018". Technical report, UNAH, Tegucigalpa: FACES-UNAH.

de Aeronáutica Civil de México, D. G. (2017). Circular obligatoria que establece los requerimientos para operar un sistema de aeronave pilotada a distancia (RPAS) en el espacio aéreo mexicano.

de Aviación Civil, A. (2018). Regulación de los vehículos aéreos no tripulados. San Salvador: AAC.

Montero, I. y León, O. G. (2002). Clasificación y descripción de las metodologías de investigación en psicología. International journal of clinical and health psychology.

Monumental, R. (2018). Radio monumental. Obtenido de Polémico reglamento para uso de drones entró en vigencia este martes: http://www.monumental.co.cr/2018/02/13/polemicoreglamentoparausodedronesentroenvigenciaestemartes/.

OACI (2019). Guía práctica oaci sobre los uas. Obtenido de Antecedentes y recomendaciones generales:https://www.icao.int/safety/UA/UASToolkit/Pages/Narrative México: DGAC MX Background_es.aspx.

República, L. (2018). Obtenido de Operadores de drones disconformes con nueva regulación: https:/ / www.larepublica.net/noticia/operadores-de-drones-disconformes-con-nueva-regulacion.

Sampieri, R., Collado, C., y Lucio, P. (2010). Metodología de la investigación. McGraw-Hill.

UNAH, U. (2017). El uso de drones será regulado en honduras. Obtenido de YOUTUBE: https://youtu.be/5dZTR9xbZ4I. 